\title{
TOPOLOGY OF LIE GROUPS
}

\section{HANS SAMELSON}

1. Introduction. The purpose of this address is to sketch some aspects of the subject known as topology of Lie groups, i.e., of the study of the topological characteristics (mainly those of homology and homotopy theory) of the underlying spaces of the Lie groups, and of the connections between topological and group theoretical properties. The interest in this field seems to stem from the fact that a variety of disciplines from algebra, analysis, and topology have found a very natural domain of application here; on the other hand, the topological study of Lie groups has resulted in contributions to other fields such as the theory of fibre bundles $[116],{ }^{1}$ (generalized) affine connections [20], metric geometry [128], and topology (one might mention that the famous theorems of de Rham [102] were first formulated in this context).

In [13] Cartan has given a beautiful account of everything that was known about topology of Lie groups at the time; in the present paper we shall try to fill in some of the subsequent developments; some overlap is of course unavoidable (see also [115; 141]). It should be said that we shall be concerned only incidentally with the general theory of topological groups; in particular we are not concerned with the developments centering around Hilbert's fifth problem, i.e., the problem of when topological groups can be proved to be Lie groups.

2. Definitions. We begin by recalling briefly basic definitions and a series of classical facts: To describe a Lie group $G$, we have first of all a manifold of some dimension $n$, i.e. a (separable) Hausdorff space, usually assumed connected, in which every point has a neighborhood which is homeomorphic with Euclidean $n$-space $E^{n}$; such a homeomorphism sets up a coordinate system in the neighborhood of the point. Secondly, the manifold carries an analytic structure: A class of coordinate systems, covering the manifold, is specified, such that wherever two of the systems overlap, one has a (real-) analytic transformation of coordinates, with nonvanishing Jacobian. It becomes then possible to introduce the concepts of (real-) analytic function,

An address delivered before the Chicago meeting of the Society, April 28, 1950, by invitation of the Committee to Select Hour Speakers for Western Sectional Meetings; received by the editors June 18, 1951 .

1 Numbers in brackets refer to the bibliography at the end of the paper. 
analytic mapping, analytic (or only differentiable) curves, vectors, usually thought of as tangent vectors to curves, the space of all vectors at a point,-which is an $n$-dimensional linear vector space (over the reals), called the tangent space at the point in question, and more generally tensors of an arbitrary index constellation. Thirdly, the manifold carries a group structure, a multiplication is defined between the points of the manifold (the product of $a$ and $b$ denoted by $a \cdot b$ or $a b$ ) satisfying the usual group axioms. Fourth, the analytic structure and the group structure are related through the requirements that the product $a b$ and the inverse $a^{-1}$ depend analytically on $a$ and $b$, i.e., the coordinates of $a b$ and $a^{-1}$ are analytic functions of the coordinates of $a$ and $b$; or expressed in terms of a mapping: the map of $G \times G$ (endowed with the natural analytic structure) into $G$ defined by $(a, b) \rightarrow a b^{-1}$ is analytic.

An important example is the group $G L(n, R)$ of all real nonsingular $n \times n$ matrices, with matrix multiplication as group operation, and analyticity defined by considering the set of all these matrices in the obvious fashion as subset of $n^{2}$-dimensional Euclidean space, and thus covering it with a single coordinate system. A second example is the group $O(n)$ of all orthogonal matrices; this is a submanifold of $G L(n, R)$, defined by the equations between the matrix elements which express orthogonality $\left(M \cdot M^{\prime}=I\right)$. Here one needs several coordinate systems to cover the group, even to cover the subgroup $O^{+}(n)$ of orthogonal matrices of determinant +1 (this is an open-and-closed connected normal subgroup of $O(n)$, of index 2); one can represent, e.g., the points in the neighborhood of the unit element $I$ by the formula $e^{S}$, with the skew symmetric matrix $S$ ranging through a neighborhood of the 0 -matrix; the elements $s_{i j}$ of $S$ with $i<j$ serve then as coordinates $[21 ; 96]$.

3. Covering groups. Homomorphisms and isomorphisms of Lie groups are always assumed to be continuous, and can then be proved to be analytic. A local homomorphism is an (analytic) map of a neighborhood of the unit element $e$ in one group into a similar set in a second group, which preserves product relations between elements in the neighborhood; local isomorphism is defined analogously. A covering group of a group $G$ is one that is mapped onto $G$ by a homomorphism, called projection, which is also a local isomorphism of the two groups; such a group is a covering space of $G$ in the usual topological sense $[108 ; 116]$. Every group $G$ possesses a unique simply connected (i.e. all closed paths are contractible to a point) "universal" covering group $G$; the-discrete-kernel of the projection is isomorphic with the fundamental group (cf. §6) of $G$. The simplest 
example is the map of the real line $R$ into the circle $S^{1}$ (complex numbers of norm 1), defined by $x \rightarrow \exp (i x)$; the kernel consists of the multiples of $2 \pi$. All groups locally isomorphic with a given group are obtained by factoring the simply connected covering group by various discrete normal (automatically central) subgroups. A local homomorphism of a simply connected group can always be extended to a homomorphism of the whole group $[107 ; 108 ; 21 ; 96]$.

4. The Lie algebra. To every Lie group $G$ there is attached the Lie algebra: let $L$ be the tangent space at $e$; let $\xi, \eta$ be two vectors of $L$, thought of as tangent vectors to two curves $x(t), y(t)$; form the commutator $x(t) \cdot y(t) \cdot x(t)^{-1} \cdot y(t)^{-1}$, divide by $t^{2}$; the limit, as $t \rightarrow 0$, is again a tangent vector at $e$, depending on $\xi$ and $\eta$, and called the commutator or Lie product $[\xi, \eta]$. This multiplication is bilinear, antisymmetric, and satisfies the Jacobi identity $[\xi,[\eta, \zeta]]+[\eta,[\zeta, \xi]]$ $+[\zeta,[\xi, \eta]]=0$, which reflects the associative law in $G$. With this multiplication $L$ is called the Lie algebra of $G$. As an example, the Lie algebra of the orthogonal group $O(n)$ consists of the skew-symmetric matrices, and $[\xi, \eta]$ is the matrix combination $\xi \eta-\eta \xi$. The principal fact concerning the Lie algebra is that two groups with isomorphic Lie algebras are locally isomorphic, and that therefore almost all properties of a group must be contained in the Lie algebra. Every subalgebra is the Lie algebra of a subgroup (which is a Lie group itself, but may or may not be closed in $G$; on the other hand, a closed subgroup is automatically a Lie group, not necessarily connected [13 (16)]); ideals correspond to normal subgroups; homomorphism of the Lie algebra and local homomorphism of the group correspond to each other. In particular, every vector in $L$ gives rise to a 1-parameter subgroup, i.e., a subgroup which is homomorphic image of the real line $R$; such a subgroup is either homeomorphic and isomorphic with $R$ (in which case it "diverges to $\infty$ ") or its closure is a torus group $T^{m}$, i.e., a product of a certain number of circle groups $S^{1}$.

The inner automorphism $x \rightarrow a x a^{-1}$ by an element $a$ leaves the unit $e$ fixed, and so induces a linear transformation $A_{a}$ (actually an automorphism) of $L$. The map $a \rightarrow A_{a}$ is called the adjoint representation, and the $A_{a}$ 's form the adjoint group; the corresponding "infinitesimal" homomorphism maps $\xi$ into ad $\xi$, where ad $\xi$ is the linear map of $L$ into itself defined by ad $\xi(\eta)=[\xi, \eta]$; it follows easily that "ideal in $L$ " is identical with "subspace of $L$, invariant under the adjoint group."

The commutator ideal $L^{\prime}$ is the ideal generated by all elements of the form $[\xi, \eta]$; it corresponds to the usual commutator subgroup. A 
Lie algebra is solvable if the sequence $L, L^{\prime}, L^{\prime \prime}=\left(L^{\prime}\right)^{\prime}, \cdots$ ends with 0 ; every Lie algebra has a unique maximal solvable ideal, the radical; a Lie algebra is semi-simple if its radical is 0 -it is then direct sum of nonabelian simple Lie algebras. A Lie algebra is simple if it has no nontrivial ideals; frequently the (one-dimensional, abelian, non-semi-simple) Lie algebra of the real line is considered not simple ex definitione. The important Levi-Whitehead theorem [135] says that any Lie algebra has a semi-simple subalgebra which maps isomorphically on the factor algebra modulo the radical, so that one has a split extension. There are of course always the corresponding definitions and facts for the groups $[21 ; 96]$.

5. Classification. A fact of very great importance is that all simple Lie algebras and groups are known (the construction of a general Lie group is reduced thereby to an-in general nontrivial-extension problem). One knows since Killing and Cartan [13 (2)] that of connected compact simple groups there are exactly the following (up to local isomorphism) $[28 ; 124 ; 138]$ : The classical groups, namely the orthogonal groups $O^{+}(n)$ for all $n>1$ (except that $O^{+}(4)$ is not simple), the groups $S U(n)$ of unitary unimodular matrices $\left(M^{*}=M^{-1}\right.$, det $M=1$ ) of degree $n$, and the symplectic groups $S p(n)$ consisting of the unitary unimodular matrices of degree $2 n$, which leave a given nondegenerate skew-symmetric bilinear form invariant; and five socalled exceptional groups $G_{2}, F_{4}, E_{6}, E_{7}, E_{8}$ (of resp. dimension 14, $52,78,133,248$ ). (One often uses the notation $A_{n}=S U(n+1)$, $B_{n}=O^{+}(2 n+1), C_{n}=S p(n), D_{n}=O^{+}(2 n)$.) They are all semi-simple except for $D_{1}$.

For noncompact semi-simple groups there is a theorem of Cartan $[13(3,14) ; 39 ; 130]$, which associates each such group uniquely with a compact group in such a way that the Lie algebras of the two groups become identical if one admits complex scalars; as an example one might cite the Lorentz group and $\mathrm{O}^{+}(4)$-with complex coordinates they become identical, since then the distinction between a definite and an indefinite quadratic form disappears.

We note here a remark of Cartan's on the development of our subject (and the theory of Lie groups in general): The early discovery of the classification has had the effect that frequently general facts about Lie groups were first so to speak "verified" for the individual simple groups, and that only later a general reason was found.

6. First topological facts. At this point topology of Lie groups proper begins, namely the study of the topological structure of the spaces which appear as manifolds of the various Lie groups. The 
interest in this question is due, besides to $\mathrm{H}$. Weyl, above all to $\mathrm{E}$. Cartan, who in a long series of papers $[13 ; 14]$ came back to the subject again and again, pointed out its importance, made a thorough study of many special cases and went on from there to prove or predict many general results.

We begin with some elementary statements about the space of our Lie group $G$ (cf. [13]):

The manifold is orientable. To show this we note that, for any fixed $a \in G$, the transformation sending $x$ into $a x$, the left translation $l_{a}$ by $a$, is an analytic homeomorphism of $G$ with itself, mapping the unit element $e$ into $a$; we choose now an orientation at $e$, and transplant it to $a$ by the left translation $l_{a}$; this orients $G$ coherently. The proof really shows more, namely that there exist vector fields without singularities on $G$, and that in fact $G$ can be "parallelized," i.e., there exist $n$ everywhere independent vector fields on $G$; we simply take $n$ independent vectors at $e$, and transplant them to the arbitrary point $a$ by $l_{a}$. It follows from topological principles [116] that all the Stiefel-Whitney characteristic classes vanish, and in particular that the Euler-Poincare characteristic $\chi(G)$ (the alternating sum of the Betti numbers, which equals the alternating sum of the numbers of cells of the various dimensions if $G$ is divided up into a complex (cf. [2])) is 0 .

The fundamental or Poincaré group $\pi_{1}(G)[109 ; 116]$, whose elements are the homotopy or deformation classes of closed paths, starting and ending at $e$, is abelian: If two such paths, $\alpha$ and $\beta$, are represented by maps $f$ and $g$ of the circle $S^{1}$ into $G$, we form the torus $T^{2}=S^{1} \times S^{1}$, and map it into $G$ by sending the point $(x, y)$ into $f(x) \cdot g(y)$. Under the induced homomorphism the generators of the fundamental group of the torus, represented by $S^{1} \times p$ and $p \times S^{1}$ ( $p$ is a suitable point of $S^{1}$ ), map into $\alpha$ and $\beta$ respectively; from the elementary fact that these two generators commute one concludes that also $\alpha \beta=\beta \alpha$.

7. Noncompact groups. We turn next to noncompact groups. Examples indicate that the noncompactness is of a trivial character: In $G L(n)$, e.g., every matrix can be expressed (through the GramSchmidt orthogonalization process) as product of an orthogonal matrix and a triangular matrix $\left(t_{i j}=0\right.$ for $\left.i<j\right)$ with positive diagonal elements. This means that $G L(n)$ is homeomorphic with the Cartesian product of the compact group $O(n)$ and a Euclidean space of dimension $n(n+1) / 2$ (the elements of the triangular matrices below the diagonal range through the whole real axis, those on the diagonal through the positive half of the real axis). Similarly, for the Lorentz 
group: any matrix can be written as product of an orthogonal and a positive definite symmetric matrix; for a matrix in the Lorentz group one shows that the two factors separately belong to the Lorentz group. That means that the Lorentz group is homeomorphic to the Cartesian product of its subgroup of orthogonal matrices and the subset of positive definite symmetric matrices; and the latter set is easily shown to be homeomorphic with Euclidean $E^{3}$. The general theorem behind this (known for simply-connected groups to Cartan [13]) is due to Malcev [80] and Iwasawa [52] (cf. also Mostow [92]): Any connected group is homeomorphic to the Cartesian product of a maximal compact subgroup $K$ (which is connected) and a subset $H$, which is homeomorphic with a Euclidean space; the theorem says more, namely, that there exist 1-parameter subgroups $h_{1}, \cdots, h_{r}$, isomorphic to the real line $R$, such that $H$ can be written uniquely as $h_{1} \ldots h_{r}$ (i.e., each element $h$ of $H$ has a unique representation $h=g_{1} \cdots g_{r}$ with $\left.g_{i} \in h_{i}\right)$ and that $G$ can be written uniquely as $K \cdot H$. Moreover all maximal compact subgroups are conjugate. The main step in the proof is to establish the theorem for the adjoint group of a semi-simple group; this is done by full use of the Lie algebra: one extends the scalars of the Lie algebra $L$ to complex numbers; in this complex Lie algebra one finds the Lie algebra $L_{c}$ of the corresponding compact Lie group (a theorem of Cartan-Weyl-Mostow [13 (14); 130; 92]); the intersection $L_{c} \cap L$ determines a compact subgroup $K$ of $G$, which turns out to be maximal, and by a careful analysis one finds a complementary subalgebra, which generates a subgroup $H$ made up of triangular matrices; $K$ and $H$ have then the required properties. For the general result, one makes use of the theorem that the decomposition is valid for a group if it is valid for a normal subgroup and the corresponding factor group; this makes possible an induction over the dimension. Practically all significant topological properties of $G$ are therefore those of a maximal compact subgroup.

8. Solvable groups. Some properties of subgroups. The special case of solvable groups is worth considering directly. Solvability is equivalent to the existence of a composition series with each factor group isomorphic with either $R$ or $S^{1}$. One proves now [52]: whenever one has a factor group $G / N$, with $N$ solvable and $G / N=R$ or $S^{1}$, then $G$ has a 1-parameter subgroup projecting isomorphically on $G / N$. Going through the composition series, one gets $n 1$-parameter subgroups $h_{1}, \cdots, h_{n}$, isomorphic with either $R$ or $S^{1}$, such that $G$ is uniquely represented as $h_{1} \ldots \ldots h_{n}$. It follows that $G$ is homeomorphic with the Cartesian product of a torus and a Euclidean space; 
this is a theorem of Chevalley $[22 ; 23]$. In particular, if $G$ is simplyconnected, it is homeomorphic with Euclidean $n$-space; this was known to Cartan long ago.

A question of Pontryagin's can be solved on the basis of the Malcev-Iwasawa theorem: If $G$ is simply-connected, and $N$ is a closed normal subgroup, is $N$ simply connected? An affirmative answer is obtained by reduction to compact groups where the result was known $[52 ; 74]$. Let $G=K \cdot H$, then $N$ and $G / N$ (the factor group) decompose according to $K \cap N \cdot H^{\prime}$ and $K N / N \cdot H^{\prime \prime}(K \cap N$ and $K N / N$ are maximal compact in $N$ and $G / N)$. $G$ is simply connected if and only if $K$ is; this in turn is so (as can be proved easily on the basis of the developments in \$10) if and only if $K \cap N$ and $K / K \cap N(=K N / N)$ are simply connected; and this in turn is equivalent to $N$ and $G / N$ being simply connected.

A last question, which goes somewhat in this direction, is the following: as mentioned, every subalgebra of $L$ determines a subgroup of $G$. When is this subgroup closed? Malcev has given invariants of the algebra, which decide the answer $[75 ; 77 ; 79 ; 80 ; 81]$; the invariants involve subspaces of $L$ with rational coefficients-the rationals enter the problem through the fact that one has to consider closed 1-parameter subgroups, and such groups on a torus correspond to straight lines through rational points in the Euclidean space which is the simply connected covering group of the torus. A useful result is that a subgroup is closed exactly if it contains the closure in $G$ of each one of its 1-parameter subgroups. Similar results have been obtained by Gotô [40]. The problem is really somewhat more complicated, because the character of the subgroup generated by a subalgebra of the Lie algebra depends also on which one in its class of locally isomorphic groups the group $G$ is. A consequence of these considerations, as shown by Mostow [93], is the fact that a semi-simple subalgebra of $L$ generates a closed subgroup if $G$ is simply connected. He also showed, under the same hypothesis on $G$, that the subgroup, generated by the subalgebra $M$, is closed if $\operatorname{dim} L$ - $\operatorname{dim} M<5$; for larger differences this is not always so.

9. Compact groups. We come now to compact groups. Compactness makes it possible to find in $L$ a positive definite quadratic form which is invariant under all transformations of the adjoint group: One takes any positive definite form and averages or integrates (with an easily defined invariant integration process $[13(16) ; 21 ; 131])$ its transforms under the adjoint transformations over the group. In other words, the adjoint group can be assumed to consist of orthogonal matrices. It follows that the Lie algebra of a compact group is com- 
pletely reducible in the sense that to every ideal (=subspace invariant under the adjoint group) there exists a complementary ideal. As a consequence, if $N$ is a closed normal subgroup, then $G$ is locally isomorphic with the direct product of $N$ and the factor group $G / N$. This means that all extensions of one compact group by another are locally isomorphic, and so they all have the same universal covering group; they are distinguished by the different discrete normal subgroups by which one has to divide. It is possible to give a complete description of the situation $[110 ; 11 ; 12]$; one can make the set of extensions of a group $N$ by a group $H$ into a group, and one has the theorem of Shapiro [110] that this group of extensions is isomorphic to the group of homomorphisms of the fundamental group of $H$ (considered as a discrete subgroup of the simply connected covering group $\widetilde{H}$ of $H$ ) into the center of $N$, modulo those homomorphisms which can be extended to all of $\tilde{H}$.

If one pursues the above reasoning somewhat further, one arrives at the fact that any compact group is locally isomorphic with the direct product of a finite number of simple groups, and more precisely is obtained from the direct product of the simply connected covering groups of these simple groups by dividing by a discrete normal subgroup.

10. Weyl's theorem. The last statement is given more precision by an important theorem of $\mathrm{H}$. Weyl, saying that the fundamental group of a compact semi-simple group is finite (or that the first Betti number is zero), in other words, that the universal covering group is still compact. There are several proofs for this theorem $[129 ; 13 ; 25$; $43 ; 105]$; a geometrical proof, which gives us occasion to introduce some useful concepts, goes along the following lines [105]: In a compact group there exists always an invariant Riemannian metricinvariant under left and right translations. The invariant quadratic form in $L(\mathrm{cf} . \S 9)$ defines the line element, the $d s^{2}$, at $e$; for any other point one gets the $d s^{2}$ by left translation; the formula $x \cdot a=a \cdot a^{-1} x a$ shows that invariance under left translations and under the adjoint group implies invariance under right translations $[13(16) ; 1]$. It turns out that the geodesics are the 1-parameter subgroups and their cosets. Now if the universal covering group (into which the metric can be lifted) is not compact, then one can find in it a straight line, i.e., a geodesic which furnishes the shortest connection between any two of its points, through $e$. By considering the relative position of this line to the kernel of the projection one can show that it must lie in the center, so that one gets a center of positive dimension which makes the group not semi-simple. A noteworthy fact about Weyl's 
theorem is that compactness for semi-simple groups appears as a property of the Lie algebra; this is of course not so for other types of groups, as the groups $S^{1}$ and $R$ show. Using Weyl's theorem it follows then easily that any compact group can actually be obtained as product of (a finite number of) compact, simply connected, simple, semi-simple groups and a torus group, modulo a finite normal subgroup $[13 ; 96]$.

11. The Betti numbers. The most obvious topological problem that arises now is the determination of the topological invariants, in particular of the Betti numbers. The results indicated in $\$ \S 9$ and 10 mean that it is sufficient to consider the simple groups of the classification given in $\$ 5$ (cf. a remark in \$16). (We shall use the language of homology or cohomology, whichever is convenient; we assume some familiarity with the concepts of algebraic topology $[2 ; 50$; $61 ; 109]$.)

The problem has been approached in various manners, some of which we shall describe briefly. The first method, Pontryagin's, amounts to a direct geometric construction of the cycles, for the classical groups [99]. Consider, e.g., in the unitary group $A_{n}$ the subgroup $A_{n-1}$, obtained by putting $a_{n+1, n+1}=1$; the (left) cosets of $A_{n-1}$ are all analytic submanifolds of $A_{n}$, homeomorphic with each other; they fill up $A_{n}$ in a very regular manner, as a matter of fact, they define a fibre decomposition [116] of $A_{n}$, i.e., a neighborhood of each coset can be mapped homeomorphically onto the Cartesian product $C^{2 n+1} \times A_{n-1}\left(C^{2 n+1}\right.$ is an open $(2 n+1)$-cell) in such a fashion that the sets $p \times A_{n-1}$ correspond to cosets of $A_{n-1}$. Pontryagin constructed now a cycle, given by a closed surface $V_{n}$ of dimension $2 n+1$, which intersects each coset in exactly one point (except the coset farthest away from $\left.A_{n-1}\right)$. The smoothness and regularity with which $A_{n-1}$ and $V_{n}$ intersect imply that their intersection number, in the sense of topology, is +1 , and that therefore, by Poincare duality, both are not homologous to zero in $A_{n}$ and so define nonzero elements of the homology groups of $A_{n}$ (with integral or rational coefficients). The same construction is now repeated in $A_{n-1}$, giving rise to a $V_{n-1}$, and so on down to $V_{1}$ (of dimension 3 ). These cycles are now combined by an operation utilizing the group property, the Pontryagin multiplication, which with two cycles $C, D$ associates the cycle $C \circ D$, where intuitively speaking $C \circ D$ is traced out by the point $p \cdot q$, if $p$ runs through $C$ and $q$ runs through $D$. (The strict definition: $C \circ D$ is the image of $C \times D$ under the map $(x, y) \rightarrow x y$ of $G \times G \rightarrow G)$. Pontryagin showed now that, in the sense of homology, the $V_{i}$ are anticommutative, and generate the whole Betti group in 
such a way that the products $V_{i_{1}} \circ \cdots \circ V_{i_{k}}, i_{1}<\cdots<i_{k}$, and the zero-cycle, given by a point, form a basis for the group. This can be expressed geometrically by saying that $A_{n}$, as far as the cycles go, behaves as if it were the product $S^{3} \times S^{5} \times \cdots \times S^{2 n+1}$ (where $S^{n}$ denotes the $n$-sphere $\left.\left\{x: x \in E^{n+1},\|x\|=1\right\}\right)$, or algebraically by saying that the Pontryagin ring (homology group with Pontryagin multiplication) is a Grassmann algebra with a unit and generators of dimension $3,5, \cdots, 2 n+1$. The intersection behavior also follows a very simple pattern, namely that given by the analogy with the sphere-product. Similar results were obtained for the other classical groups. We describe the results by introducing the Poincarépolynomial of a space, the polynomial in which $t^{k}$ has for coefficient the $k$ th Betti number. We have then

$$
\begin{aligned}
& P_{A_{n}}=\left(1+t^{3}\right)\left(1+t^{5}\right) \cdots\left(1+t^{2 n+1}\right), \\
& P_{B_{n}}=\left(1+t^{3}\right)\left(1+t^{7}\right) \cdots\left(1+t^{4 n-1}\right), \\
& P_{C_{n}}=\left(1+t^{3}\right)\left(1+t^{7}\right) \cdots\left(1+t^{4 n-1}\right), \\
& P_{D_{n}}=\left(1+t^{3}\right)\left(1+t^{7}\right) \cdots\left(1+t^{4 n-5}\right) \cdot\left(1+t^{2 n-1}\right) .
\end{aligned}
$$

12. Differential forms. The second approach to the Betti numbers, initiated by Cartan and completed by $R$. Brauer, is algebraic in character $[9 ; 13]$. It starts from a different interpretation of the Betti numbers: By the famous theorem of de Rham [102] (which was inspired by just these questions) the $p$ th Betti number is equal to the number of linearly independent $p$-dimensional closed exterior differential forms defined over $G$ modulo those forms which are derivatives of $(p-1)$-forms. (The value or expression of such a form at any point is a skew-symmetric covariant tensor with $p$ indices or, in another description, a skew-symmetric function $\omega\left(\xi_{1}, \cdots, \xi_{p}\right)$ of $p$ arbitrary tangent vectors of the point, linear in each variable; the derivative referred to is the exterior derivative of the form or the alternating derivative of the tensor; closed means with derivative 0 ; cf. $[21 ; 50]$ and ref. 64 in $[14]$; we use the symbol $d$ for derivation.) Now one can reduce the $p$-forms by integrating or averaging over the group to bi-invariant forms, i.e., to forms which are invariant under left and right translations. (Incidentally, the bi-invariant forms turn out to be identical with the harmonic forms, relative to the invariant metric of the group, and can be treated from this point of view [50].) A theorem of Cartan's [13 (15); 25] (an easy consequence of the Maurer-Cartan equations, which state that for left-invariant 1-forms the relation $d \omega(\xi, \eta)=-\omega([\xi, \eta])$ holds at $e)$ says that a bi-invariant form is automatically closed; and this means that the $p$ th Betti 
number is equal to the number of linearly independent bi-invariant forms (modulo nothing). A bi-invariant form is determined by its expression at $\boldsymbol{e}$ (the expression at any other point is obtained by left translation), and there it has to be invariant under the adjoint group (cf. the case of the metric in $\$ 10$ ). The pth Betti number is therefore equal to the number of linearly independent skew-symmetric tensors with $p$ indices, at $e$, invariant under the adjoint group; and the problem of finding the Betti numbers is translated into a problem in representation theory. (One sees by the way that the $p$ th Betti number can be at most $C_{n, p}$, this being the maximum number of independent skew $p$-tensors over a linear space of dimension $n$.) By use of the first main theorem of invariant theory, Brauer was able to construct, for each classical group, explicitly a certain number of invariants, which from other considerations could be shown to exhaust all possible invariants (cf. also $[130 ; 131])$. The result corresponded completely to Pontryagin's, i.e., certain basic forms $\omega_{1}, \omega_{2}, \cdots$ were found, such that the products $\omega_{i_{1}} \cdot \omega_{i_{2}} \cdots \cdots \omega_{i_{k}}$ with $i_{1}<i_{2}<\cdots<i_{k}$ and the scalars constitute a basis for the group of invariant forms. The whole method of invariant differential forms is due to Cartan; he had, before Brauer gave the complete result, obtained certain facts about the Betti numbers, enough to make him state as a conjecture what then turned out to be the correct result [13 (15)].

13. Hopf's theorem. The next approach, due to Hopf, differs from the first two by establishing a general theorem instead of considering each group separately $[45 ; 46]$. The method is topological and starts from the map $\phi$ of $G \times G$ into $G$, given by $\phi(x, y)=x \cdot y$. The existence of the unit element $e$ means that every cycle of $G \times G$, which "lies in one of the two factors," i.e., which is of the form $z \times e$ or $e \times z$ with some cycle $z$ of $G$, is mapped by $\phi$ into $z$; for the associated or adjoint map $\phi^{*}$, which maps the cocycles (or differential forms, if one prefers) of $G$ into those of $G \times G$, this means that $\phi^{*}(a)$ is of the form $a \times 1+1 \times a+R$, where 1 is the unit cocycle and $R$ consists only of "mixed terms" $c \times d$, where both factors have positive dimensions. Now one draws algebraic conclusions. To illustrate, we show that a sphere of even dimension cannot be a Lie group (of course this follows also from the characteristic). If $t^{n}$ is the basic $n$-cocycle of $S^{n}$, then one would have $\phi^{*}\left(t^{n}\right)=t^{n} \times 1+1 \times t^{n}$ (since there are no "in between" terms in the ring of $\left.S^{n}\right) .\left(t^{n}\right)^{2}$ is 0 , for dimension reasons. $\phi^{*}$ being a homomorphism, the square of $t^{n} \times 1+1 \times t^{n}$ has to vanish; under the sign-rules in a Cartesian product, remembering that $n$ is even, one obtains $2 t^{n} \times t^{n}$ for the square, which is not 0 , and thus has a contradiction. Refining this 
argument considerably one obtains the theorem: The cohomology ring of a Lie group contains certain "primitive" elements $a_{1}, \cdots, a_{l}$ (of odd dimension and therefore anticommutative), such that the products $a_{i_{1}} \cdots a_{i_{k}}, i_{1}<\cdots<i_{k}$, and the unit 1 form a basis for the cohomology group; the ring is isomorphic with the Grassmann algebra over the linear space spanned by the $a_{i}$. Any such set of elements $a_{i}$ is called a set of generators of the ring (strictly speaking one would have to add the unit to this set). The number $l$ of elements $a_{i}$ has a group theoretical meaning, which we discuss in $\$ 20$. One can show in addition that the homology group, with Pontryagin multiplication, is also a Grassmann algebra, and that these two Grassmann algebras are duals of each other $[59 ; 62 ; 104]$. While thus the theorem gives a reason for the specific structure of the cohomology rings of the classical groups as found by Pontryagin and Brauer, it does not of course determine them explicitly; for the classical groups, e.g., one can couple Hopf's theorem with a theorem about transitive transformation groups of spheres (to which we shall come later) to arrive at the Betti numbers.

14. Cohomology theory of Lie algebras. The fourth method works with the Lie algebra. By the integration process mentioned above, one reduces the differential forms (cf. \$12) to forms which are invariant under left translations (invariance under right translations, as in $\$ 12$, is not required here); such forms are determined by their expression at $e$; moreover the process of taking the exterior derivative can be translated into a simple algebraic operation in the Lie algebra (the Maurer-Cartan equations, $\$ 12$, show how this translation goes for 1-forms; the complete formula, which follows easily from this, is one of a series of algebraic "coboundary formulae," which recently have become popular, and reads $d \omega\left(\xi_{1}, \cdots, \xi_{p+1}\right)$ $=\sum_{i<j}(-1)^{i+i} \omega\left(\left[\xi_{i}, \xi_{j}\right], \xi_{1}, \cdots, \hat{\xi}_{i}, \cdots, \hat{\xi}_{j}, \cdots \xi_{p+1}\right)$; the "roofs" mean that the vectors are to be omitted). The whole cohomology theory of differential forms is so expressible by simple algebraic operations in the Lie algebra, and the problem arises, to prove topological facts about groups on this basis [25]. Koszul proved Hopf's theorem and more in this manner [59]. The development naturally parallels somewhat that in Hopf's theory. Instead of the product $G \times G$ there appear algebraic constructions like $L \times L$, the direct product of the Lie algebra with itself; instead of the natural map of $G \times G$ into $G$ there appears the natural map of $L$ onto the diagonal of $L \times L$; instead of the forms or cochains there appears the Grassmann algebra $A$ over $L$. Hopf's theorem becomes the statement that the cohomology ring of a Lie algebra is a Grassmann algebra generated by certain "primi- 
tive" elements. It is formulated and proved for any field of characteristic 0 as coefficient domain, under assumptions on $L$ which replace the condition of compactness on $G$.

15. The exceptional groups. Symmetric forms. The five exceptional groups have resisted much longer (or rather four of them; $G_{2}$ was known for some time [13 (15)]). Recently Yen determined their Betti numbers [139], using methods which involve knowledge of the representations of the groups (somewhat in the spirit of \$12) and many additional pieces of information, in particular a conjectured formula of G. Hirsch about fibre bundles, when bundle and fibre have the homology structure of products of spheres (as Lie groups do, by Hopf's theorem). The dimensions of the primitive cocycles for the five groups are: $(3,11),(3,11,15,23),(3,9,11,15,17,23)$, $(3,11,15,19,23,27,35),(3,15,23,27,35,39,47,59)$. In the meantime Chevalley and Weil [26] have found a totally different approach to the whole problem, which made the computations for all groups much simpler. The leading idea is the introduction of symmetric, invariant (under the adjoint group) forms in $L$ instead of skew-symmetric ones; by a process which involves substitution of commutators $[\xi, \eta]$ for the elements in a symmetric form, one can associate with each symmetric $p$-form a skew form (of degree $2 p-1$ ) (a special case is familiar: from the symmetric bilinear form, introduced by Cartan, $\sigma(\xi, \eta)(=\operatorname{tr}(\operatorname{ad} \xi$ ad $\eta))$, one obtains in $\sigma(\xi,[\eta, \zeta])$ a skew 3 -form $\psi(\xi, \eta, \zeta))$. A crucial theorem states that the primitive elements of the cohomology ring can be so obtained. The invariant symmetric forms are closely related to the generalized Casimir operators of Racah [101]; in particular the degrees of these operators for the exceptional groups, as given by him, turn into the dimensions of the primitive cocycles upon replacing $p$ by $2 p-1$.

16. Torsion. Besides the Betti numbers there are also the torsion coefficients to determine, or in other words, the elements of finite order in the cohomology group with integral coefficients. It is well known here that the fundamental group of the rotation group of 3 -space is of order two; actually this is so for all orthogonal groups $O^{+}(n), n>2$ [116]. Pontryagin has shown in his explicit construction [99] that the unitary and symplectic group have no torsion at all, and that all the torsion elements of the orthogonal group are of order two. The Poincaré polynomial $\bmod 2$ of $O^{+}(n)$ is given by $(1+t)$ $\cdot\left(1+t^{2}\right) \cdots \cdot\left(1+t^{n-1}\right)$. It has recently been shown by A. S. Shapiro and A. Borel, using the topological theory of sphere-bundles (in particular the theory of the characteristic cocycle) and Leray's homol- 
ogy theory that the simply connected covering groups of the orthogonal groups also have torsion (from some $n$ on). A general study of the problem of torsion in groups and also in homogeneous spaces (cf. $\$ 17$ ), based on the theories of H. Cartan, Koszul, Leray, Weil (cf. \$22), has been made by A. Borel [9]. Among other things, he has determined completely the torsion of the universal coverings of the orthogonal groups, and of some of the exceptional groups. The cohomology mod 2 for the orthogonal group and related spaces has been investigated by C. E. Miller [86]. (That the Betti numbers of a compact group and any compact covering group are the same is easily proved by interpreting the Betti numbers via bi-invariant forms: both groups have the same adjoint group. The same device shows that for a compact group $G$ and connected normal subgroup $N$ the "Künneth relation" $P_{G}(t)=P_{N}(t) \cdot P_{G / N}(t)$ holds between the Poincare polynomials.) As special facts we mention that for a compact semi-simple group the second Betti number is always zero and that the third is always positive [13 (15)] (the invariant form $\psi(\xi, \eta, \zeta)$ mentioned in $\$ 15$ is nonzero on such groups; incidentally this proves that of the spheres $S^{n}$ only $S^{1}$ and $S^{3}$ are groups [103]). Koszul recently proved from the Lie algebra, making use of the connection between symmetric 2 -forms and skew 3 -forms mentioned in $\$ 15$, that for a simple Lie group (except the 1-dimensional $D_{1}$ ) the third Betti number is always one $[54 ; 59]$; this checks of course with the explicit results. We also note an isolated fact about noncompact simple groups: as shown by Cartan, the first Betti number is 0 or 1 $[13 ; 13(16)]$. The distinction between the two cases is related to the question of the existence of a faithful representation [13]; this question has been investigated by Gotô $[40 ; 41]$ and Malcev [78] (cf. also $[3 ; 4 ; 42 ; 43 ; 83]$ ).

17. Klein spaces. Closely related to Lie groups is another class of spaces, the Klein spaces. There are two equivalent definitions: as coset spaces and as homogeneous spaces [141]. If $H$ is a closed subgroup of the Lie group $G$, then the left cosets of $H$ can be considered as the points of a new space, the coset space $G / H$, with the customary decomposition topology [2, p. 63] (if $H$ is normal, this is the factor group); the Lie property of $G$ and $H$ imply that $G / H$ is an analytic manifold, whose dimension is the difference of those of $G$ and $H$; the natural map $p$ of $G$ into $G / H$, which sends $g$ into the coset $g H$, the projection, is analytic. A homogeneous space is a space (which we assume to be a manifold) $W$, on which $G$ acts transitively as a transformation-group, i.e. each $g \in G$ determines a homeomorphism $T_{q}$ of $W$ with itself, such that $T_{g_{1}} \cdot T_{g_{2}}=T_{g_{1} g_{2}}$, that $T_{g}(x)$, or in short 
$g(x)$, depends continuously on $g$ and $x$, and that for any $x$ and $y$ in $W$ there is a $g$ in $G$ with $T_{\theta}(x)=y$.

The connection between the two concepts is as follows: If $G$ acts on $W$, let $x_{0}$ be a point of $W$, and consider the closed subgroup $H$ $=G_{x_{0}}$, consisting of those elements of $G$ which leave $x_{0}$ fixed (it is called the isotropy or stability group at $x_{0}$ ). Each left coset of $G_{x_{0}}$ is identical with the set of elements of $G$ which send $x_{0}$ into one and the same point $x$, and conversely for each point $x$ the set of elements which map $x_{0}$ into $x$ is a left coset of $H$; one has thus a 1:1 correspondence between $W$ and $G / H$, which turns out to be a homeomorphism. (The concepts make sense for any topological group; but in general one can assert only that the map from $G / H$ to $W$ is continuous.) In particular, $W$ can be made into an analytic manifold, and $G$ acts analytically on it. As an example, the group $O^{+}(n+1)$ acts transitively on the unit sphere $S^{n}$ in $E^{n+1}$; the isotropy group is simply $\mathrm{O}^{+}(n)$. Other examples of Klein spaces are the groups themselves (with $H=\{e\}$ ), the projective spaces, the complex projective spaces $K$ (one can take $S U(n+1)$ as group $G$ ), the Grassmann-manifolds $M_{n, k}$, formed by all planes of dimension $k$ through the origin of $E^{n}$, with the orthogonal group $O(n)$ as $G$. There arises the problem of the relations between the topological properties of $G, H$, and $G / H$. Actually $G$ is a fibre bundle over $G / H$ (with $G / H$ as base space), with fibre $H$; i.e., the cosets $x H$ fill out $G$ in such a regular arrangement that each coset has a neighborhood which is homeomorphic with $C \times H$ (where $C$ is a cell of dimension equal to that of $G / H$ ) in such a fashion that the sets $p \times H$ correspond to the cosets of $H$ (cf. $\$ 11)-G$ is "locally a Cartesian product"; one should add that the structure group of the bundle is $H$ [116]. This fact is quite important; a good part of our knowledge of coset spaces was obtained by the application of fibre bundle methods. (Conversely the homology theory of Lie groups has recently found applications to the theory of fibre bundles and the related field of differential geometry [20]; even knowledge of the explicit form of the cohomology ring of, e.g., the orthogonal group, has proved useful.)

A slight generalization of the above is worthwhile: if $H$ and $K$ are two subgroups with $H \subset K$, then $G / H$ is a fibre bundle over $G / K$ with fibre $K / H$; the projection or fibre map is the natural one, which sends each coset of $H$ into the coset of $K$ containing it. If, e.g., $H$ is $K_{0}$, the component of $e$ in $K$, then $K / K_{0}$ is discrete, and $G / K_{0}$ is simply a covering space of $G / K$, with the number of sheets equal to the number of components of $K$. This has some consequences for the fundamental group of a Klein space: If $K$ is connected, then $\pi_{1}(G / K)$ 
is easily seen to be a factor group of $\pi_{1}(G)$ and therefore abelian; in the general case it has $\pi_{1}\left(G / K_{0}\right)$ as abelian normal subgroup, with index equal to the cardinality of $K / K_{0}$ (cf. [109]).

As to orientability, one can show by an argument similar to one used for groups in $\$ 6$ that a Klein space is orientable if and only if all the transformations of the isotropy group at a point $x$ are orientation preserving at $x$ (i.e., of index +1 or of positive Jacobian at $x$ ).

18. Compact Klein spaces. Characteristic. A first result, only recently established by Montgomery [91], is the following: If a Klein space, with connected isotropy group, is compact, then it is coset space of a compact group. One begins by representing $G$ and $H$ as $E \cdot K$ and $E^{\prime} \cdot K^{\prime}$ from the Malcev-Iwasawa theorem ( $\left.\$ 7\right)$, where $E$ and $E^{\prime}$ are homeomorphic with Euclidean spaces, and $K$ and $K^{\prime}$ are maximal compact subgroups (the interchange of the factors against $\$ 7$ is of course possible; we use this order now, because we use left cosets). One can assume $K^{\prime} \subset K$. It is possible then to write $G / H=\left(G / K^{\prime}\right) /\left(H / K^{\prime}\right)=E \cdot\left(K / K^{\prime}\right) / E^{\prime}$ with an (it is hoped) obvious symbolism (cf. §17). Now if the fibre is contractible to a point (as $E^{\prime}$ is), it is known [116] that there exists a cross section, i.e., a map $f$ : $W \rightarrow E \cdot K / K^{\prime}$, where $f$ followed by the projection is the identity. Since $E$ is also contractible, one can $\operatorname{deform} f$ into $f^{\prime}$, where $f^{\prime}$ maps $W$ into $K / K^{\prime}$ (into the "slice" of $E \cdot K / K^{\prime}$ at $e$, parallel to the second factor). The identity of $W$ being an essential map ( $W$ is a compact manifold), it follows that the composition of $f^{\prime}$ with the projection still maps onto $W$, and that therefore the projection maps $K / K^{\prime}$ onto $W$, which means that $K$ is transitive over $W$, q.e.d.

We consider now compact Klein spaces, with compact group. As noted in $\$ 6$, the characteristic of the group is zero; this came from the - trivial-existence of a vector field, and the-nontrivial-relation between vector fields and characteristic. For Klein spaces there is the theorem (with a vaguely parallel proof) that the characteristic is non-negative $[49 ; 129]$. The distinction between 0 and $>0$ is group theoretical, as follows: an abelian subgroup of maximal dimension of the group is known to be a torus of a certain dimension $l(G)$, called the rank of $G$ (all maximal toral subgroups are conjugate to each other); the characteristic of $G / H$ is positive exactly if $l(G)$ $=l(H)$. The basis of the proof is the consideration of $G / T$, where $T$ is a maximal toral subgroup (all other cases can be reduced to this). It is relatively easy to show (the trivial part) that there are elements of $G$ which transform $G / T$ with only a finite number of fixed points, each of index $(-1)^{m}$ (where $m=\operatorname{dim} G / T$ ); and then one reads off from the Lefschetz fixed point formula (the nontrivial part) [2] that 
the characteristic equals the number of fixed points and is therefore non-negative. As an example, consider the sphere $S^{2}$; any rotation with a finite number of fixed points has exactly two of them, and the characteristic is well known to be two. Actually, all the transformations of $G / H$ are isometries with respect to a suitable Riemannian geometry; and the theorem should have a connection with the generalized Gauss-Bonnet formula, which expresses the characteristic as integral of an $n$-dimensional curvature (cf. [18] and the references there), in other words, one should be able to show that for Klein spaces this curvature is non-negative. To illustrate the meaning of the theorem, consider Riemann surfaces of genus $p>1$, i.e., of negative characteristic. It is known that they do not admit 1-parameter groups of conformal mappings, and also that the periods of their periodic homeomorphisms are bounded [115], so that they are about as far from being Klein spaces as one can wish.

19. The diagram. We digress at this point to describe a construction, which goes back to Cartan and Weyl, which is of fundamental importance for the problem of classifying the simple Lie groups and without which no report on Lie groups would be complete. If, as above, $T^{l}$ denotes a maximal toral subgroup of the compact Lie group $G$, then one sees easily that the normalizer $N_{T}$ consists of a finite number of cosets of $T^{l}$ (the reason for the finiteness is the fact that the automorphisms of a torus form a discrete group, and that therefore any element of $N_{T}$, which is close enough to $e$, commutes elementwise with $T^{l}$, and so belongs to it). Conjugation of $T^{l}$ with elements of $N_{T}$ determines then a finite group $\Phi$ of automorphisms of $T^{l}$. A basic fact, which we shall naturally not prove here, is that this group, as transformation group of the torus $T$, determines the Lie algebra of $G$ [121]. The group $\Phi$ is easier to see if one considers the universal covering Euclidean space $E^{l}$ of $T^{l}$; there $\Phi$ turns into a discontinuous group $\Gamma$ of orthogonal transformations (rigid motions) by adding to the rotations given by $\Phi$ the covering transformations (deck transformations) of $E^{l}$ over $T^{l}$ (which are translations of $\left.E^{l}\right)$.

The group $\Gamma$ is generated by reflections in certain (infinitely many of course) planes; the planes form the so-called diagram of the group [121]. The fundamental domains of $\Gamma$ are convex polyhedra, which are all congruent to each other, and cover $E^{l}$ without overlapping; the angles between two faces of a polyhedron are of the type $\pi / k, k$ integral and not less than 2 . If the group is simple, the polyhedron is a simplex, and the determination of the simple groups is reduced to the discussion of simplices satisfying the arithmetical condition on 
the angles. The relation of this polyhedron to the group might be better understood if one remembers that all maximal toral subgroups are conjugate to each other, that therefore each element of $G$ has a conjugate on $T^{l}$, and that two elements of $T^{l}$ are conjugate in $G$ if an operation of $\Phi$ maps one into the other; finally, therefore, the polyhedron in $E^{l}$ plays the role of a fundamental domain of $G$ with respect to conjugation. The elements corresponding to points on the faces of the polyhedron, and their conjugates, have normalizers of dimension greater than $l$, actually even greater than or equal to $l+2$; such elements are called singular. The singular elements form a set of dimension at most $n-3$ (transforms of an (l-1)-dimensional set with at most $(n-(l+2))$-dimensional orbits). One can conclude from this construction that, if $G$ is simply connected, the set of nonsingular (regular) elements can be contracted over itself into the point $e$, along 1-parameter subgroups $[13(10,11) ; 121 ; 122 ; 130 ; 131]$. A new application of these ideas has been given by Stiefel: He has devised an approach, based on the diagram of $G$, to the problem of finding the Betti numbers, which yields, e.g., once more the Betti numbers of the classical groups [123].

20. The rank. The maximal toral group $T$ also plays a role for the group-theoretical interpretation of the number $l$ of generators of the cohomology-ring of $G$ (cf. $\$ 13$ ); the simple result is that $l$ equals the dimension of $T$ (=the $\operatorname{rank}$ of $G$ ). The proof employs the "power" map $p_{k}$, which sends $x \in G$ into its $k$ th power $x^{k}$ for integral $k \neq 1$. From the homology properties of $G$, as described in $\$ 13$, one can show that the (homology-) degree of $p_{k}$ is $k^{l}$ (if we denote by $G$ also the basic $n$-cycle on the $n$-manifold $G$, then we have $\left.p_{k}(G)=k^{l} \cdot G\right)$. On the other hand, if one takes a "general" element $a$ of $T$ (i.e., one whose coordinates are independent $\bmod 1$, so that its powers are dense in $T$ ), one can see by an elementary consideration that there are exactly $k^{l(\theta)}$ elements on $T$ which map into $a$ under $p_{k}$, and that no other elements of $G$ can map into $a$. In addition, one can show that the functional-determinant of $p_{k}$ (with respect to a given orientation of $G$ ) is always non-negative, and for the elements under consideration even positive; this means that the index of $p_{k}$ of all the elements which map into $a$ is +1 , and that the degree of $p_{k}$ at $a$ is $k^{l(\theta)}$. Applying now the theorem that the homology degree of a map equals its degree at any point [2], one finds $k^{l}=k^{l(G)}$, and so $l=l(G)[47]$.

21. The subgroup as a cycle. We return now to the discussion of a Klein space $G / H$. The submanifold $H$ of $G$ can be considered as a cycle, in the sense of topology, in $G$. As such it either bounds or it doesn't bound; this turns out to be an important distinction. The 
case of $H$ not bounding is by far the simpler (incidentally, no cycle of $H$ can then bound in $G$, unless it bounds already in $H$-a very special behavior [104]), and one has there a very precise result on $G / H: G$ behaves homologically (over the rationals) as if it were the Cartesian product of $H$ and $G / H$ (in general it does not actually factor); the cohomology ring of $G / H$ is also a Grassmann algebra, with generators of odd dimensions, and the ring of $G$ is isomorphic with the tensor product of the rings of $G / H$ and $H$. These two facts completely determine the ring of $G / H$ in terms of those of $G$ and $H$ : if from the generators of the ring of $G$ one drops those which correspond to generators of $H$, then the remaining ones generate a ring isomorphic with that of $G / H$. This has been proved by the topological and algebraic methods discussed in $\$ \$ 13$ and 14 , suitably extended to the case of Klein spaces $[59 ; 104]$. For the topological proof one considers the natural map of $G$ onto $G / H$. The principal fact in the proof is that the assumption on $H$ implies that the Betti groups of $G$ are mapped onto those of $G / H$; the remainder of the proof consists in a discussion of the behavior of the projection with respect to Pontryagin multiplication and the connection with the induced cohomology map. A particular case where this applies is the case where $G / H$ is a sphere of odd dimension; this is one of the theorems needed for the determination of the Betti numbers of the classical groups as mentioned in $\$ 13$ (the classical groups appear in a natural way as transitive transformation groups of spheres; the isotropy groups are again classical, and an induction becomes possible). Of course, one has to prove first that in this case $H$ is not homologous to zero; but that is easy, with a construction to be described in a moment. The remaining case of a subgroup $H$, which is $\sim 0$ in $G$, is not completely understood, and much remains to be done. A special case of interest in connection with the problem just mentioned is known, namely when $G / H$ is a sphere $S^{m}$ of even dimension; the result is that one of the primitive generators of the ring of $H$ has dimension $m-1$, and that the ring of $G$ is obtained by replacing this generator by one of dimension $2 m-1$. The topological proof employs again the map $p$ from $G$ to $G / H=S^{m}$. The principal fact (for odd and even $m$ ) is that $S^{m}$ can be lifted back into $G$ "with one singularity"; more precisely one can find an $m$-cell $C^{m}$ in $G$, such that the boundary of $C^{m}$ lies in $H$ and the projection $p$ maps the interior of $C^{m}$ topologically on the sphere $S^{m}$ minus the point which is represented by the coset $H$ itself ( $p$ maps the whole boundary of $C^{m}$ into this point). One can show now that the boundary of $C^{m}$, which is an $S^{m-1}$ lying in $H$, is $\sim 0$ in $H$ for odd $m$, from which it follows 
easily that then $H$ is not $\sim 0$ in $G$ (by a consideration involving Poincaré duality as in $\$ 11$; one starts by completing $C^{m}$ to a cycle by means of a chain in $H$ ); for even $m$ this $S^{m-1}$ is not $\sim 0$ in $H$, and as a matter of fact it is a primitive $(m-1)$-cycle in $H$ (which is $\sim 0$ in $G$, since it bounds the cell $\left.C^{m}\right)[104]$.

22. Algebraic methods. A recent result of Koszul generalizing a result of $\$ 21$ can be described roughly as follows: For every primitive $p$-cycle of $H$ which bounds in $G$, there exists a $(p+1)$-dimensional generator in the cohomology ring of $G / H$. It gives thus a first indication of what the structure of the ring of $G / H$ might be in the general case. Koszul's methods [59], which operate with the Lie algebra of $G$ and a subalgebra corresponding to $H$ (the "relative case" of \$14), are inspired by the concepts of ring of a map and of spectral ring of a filtered differential algebra, which Leray has recently introduced into topology and which have found an increasing number of applications in the recent past; it is impossible to indicate definitions or proofs here. We only mention a concept which also plays a role elsewhere, that of transgression; in terms of differential forms, it is defined as follows: If $\omega$ is a closed form in $G / H$ whose image $p^{*} \omega$ (under the map $p^{*}$ associated with the map $p$ of $G$ into $G / H$ ) is homologous to zero in $G$, i.e., $p^{*} \omega=d \alpha$, then $\alpha$ induces on the submanifold $H$ of $G$ a closed form, which is said to be transgressive. This process can be considered as the dual of the process of finding cycles in $G / H$ which are $p$-images of chains of $G$, whose boundaries are not necessarily 0 , but are collapsed into 0 by $p$ (a special case occurred in \$21). A main result of the theory is that just the primitive elements are transgressive. Leray himself has announced a number of applications of his ideas [62-73]. He has determined, for the classical groups, the ring of $G / T$, where $T$ is a maximal toral subgroup of $G$, and more generally the ring of $G / H$, when $H$ has the same rank as $G$, i.e., when it contains $T$. The results [69] are best stated in terms of the Poincaré polynomials: If $G$ and $H$ have respectively the polynomials $\Pi\left(1+t^{2 m_{\lambda}-1}\right), \Pi\left(1+t^{2 n_{\lambda}-1}\right)$, then $G / H$ has the polynomial $\Pi\left(1-t^{2 m} \lambda\right) /\left(1-t^{2 n \lambda}\right)$. That this formula holds if, instead of the groupsubgroup structure, one has only a fibre bundle is Hirsch's conjecture (\$15); the case where $G$ and $H$ are spheres, so that the products contain only one factor, is one of Gysin's results (cf. ref. 38 in [116]).

Another result in this direction was given by Koszul [56] for the case where $H$ is a circle group $S^{1}$, where therefore $G$ is fibred into circles; it states that the Poincaré polynomial of $G / H$ is obtained from that of $G$ by replacing a factor $1+t^{3}$ by a factor $1+t^{2}$. A trivial 
example for this is the group $S U(2)$, which is homeomorphic with $S^{3}$, and for which $G / S^{1}$ is homeomorphic with $S^{2}$; the interest of the result comes of course from all cases but this one.

Considerable advances along these lines have been made recently by H. Cartan [16; 17], Koszul [60], and Leray [71]. The introduction of symmetric forms (=symmetric multilinear functions on the Lie algebra, cf. §15) has proved very fruitful. To mention one of the main results, let $I_{S}(G)\left(I_{A}(G)\right)$ denote the algebra of invariant (under the adjoint group) symmetric (skew-symmetric) forms of the group $G$. For a subgroup $H$ of $G$ the Lie algebra of $H$ is contained in that of $G$; one has therefore a natural map of $I_{S}(G)$ into $I_{S}(H)$, by restriction of the arguments of the forms. This mapping completely determines the cohomology ring of $G / H$; the ring is constructed by suitable definition of a boundary operator in the tensor product $I_{A}(G) \otimes I_{S}(H)$. As a more specific result it has been found that a formula of the Hirsch type holds not only in the case where $G$ and $H$ have equal rank (cf. above), but also for symmetric spaces $G / H$ (cf. \$24); in this case the denominator polynomial can contain fewer factors than the nominator. A concept that has evolved in these developments is that of the Weil algebra $W(L)$ of a group $G$ (or rather of the Lie algebra $L$ of $G)$; it is the tensor product of the Grassmann algebra $A(L)$ of skew forms on $L$ and the algebra $S(L)$ of symmetric forms on $L$. The Weil algebra turns out to be the algebraic equivalent of a classifying space (for arbitrary dimension) for principal fibre bundles with fibre $G$ (cf. §30). What corresponds to the cohomology ring of the base space is just $I_{s}(L)$ (with all degrees doubled). The notion of infinitesimal connection, after being given a very algebraic formulation, makes it possible to define, for a principal fibre bundle with fibre $G$ and base space $B$, a homomorphism of $I_{S}(L)$ into the cohomology ring of $B$ [the image is the so-called characteristic ring of the bundle (cf. \$30)]; and one can give an algebraic construction for the full ring of the bundle from the connection.

Many of these and many other results, in particular for fields of arbitrary characteristic as coefficients (i.e., roughly speaking for the torsion group, cf. §16), have been obtained by A. Borel $[9 ; 9 \mathrm{a}-9 \mathrm{e}]$, utilizing Leray's theory.

23. Grassmann manifolds. We come now to what can be called special results, namely results on explicit spaces or classes of spaces. The first results here are due to Ehresmann [32-37], on the Grassmann manifolds (cf. \$17) and related spaces. The procedure is very direct; the space is decomposed into cells, and from the cell structure the invariants are calculated. The cells in question are of course not 
arbitrary, but related to the algebraic-geometric properties of the space. For the Grassmann manifolds, e.g., the cells are defined by the so-called Schubert varieties $[34 ; 35]$ : In Euclidean $n$-space $E^{n}$ we consider a fixed increasing sequence $E^{1} \subset E^{2} \subset \cdots \subset E^{n-1} \subset E^{n}$ of subspaces (through the origin of $E^{n}$ ). Given now a sequence of integers $a_{i}, i=1, \cdots, k$, such that $0 \leqq a_{1}<\cdots<a_{k} \leqq n-k$, we denote by $\left[a_{1}, a_{2}, \cdots, a_{k}\right]$ the set consisting of all $k$-dimensional subspaces $L$ (through the origin) of $E^{n}$ which have the property that $\operatorname{dim} L \cap E^{a_{i}+i} \geqq i$, for $i=1, \cdots, k$. This set is a (pseudo-) manifold of dimension $\sum_{1}^{k} a_{i}$; it is a generalized cell in the sense that it is obtainable from a Euclidean cell of the same dimension by making certain identifications on the boundary. All the cells so defined form a (generalized) cell decomposition of the Grassmann manifold $M_{n, k}$. The boundary relations (in the sense of combinatorial topology) have been determined by Ehresmann; the Betti numbers and torsion coefficients can then be computed. Going further in this direction, Chern [19] has determined the cohomology ring structure of $M_{n, k}$ (with coefficients mod 2); the interest in this ring is due to the fact that it is a universal antecedent of the so-called characteristic ring of any sphere bundle (cf. $\$ 30$ ). (See also [100a; 100b] in this connection.)

Similar methods have been used to determine the homology invariants of the quadrics (real, complex, quaternion, hermitian) and similar spaces $[32-37 ; 95 ; 118]$.

A different cell decomposition, related to one used by J. H. C. Whitehead [136], has been employed by C. E. Miller [86] to investigate in detail the cohomology ring mod 2 of the orthogonal group and of several of its coset spaces, e.g. the Stiefel manifolds (cf. §30). The cells here are essentially products of projective spaces. It turns out that, while the Pontryagin ring mod 2 is still a Grassmann algebra (in particular the squares of the generators vanish) this is not so for the cohomology ring mod 2; the defining relations state that certain higher powers of the generators vanish. (In the simplest case, $\mathrm{O}^{+}(3)$, which is projective 3 -space, one has one generator $x$ and the relation $x^{4}=0$.) In addition, the Steenrod squares (operations in the cohomology ring, recently introduced by Steenrod) are determined for these spaces, with an application to the problem of vector fields on the $n$-sphere (cf. $\$ 30$ and [119]).

24. Symmetric spaces. A second method, also initiated by Ehresmann [32] and perfected by Iwamoto [51] applies to the symmetric Riemannian spaces; these are spaces which have the property that for each point the symmetry, i.e. the transformation which reverses 
all geodesics through the point, is an isometry. Obvious examples are Euclidean spaces and spheres. Cartan has shown [13 (16)] that the symmetric spaces are exactly the coset spaces $G / H$, where $G$ has an automorphism of order two, which leaves just the elements of $H$ (or rather of the component of $e$ in $H$ ) fixed, and where $H$ is compact. As a nontrivial example let $G=S U(n)$ and let the automorphism be complex conjugation; $\mathrm{H}=\mathrm{O}^{+}(n)$ consists then of the orthogonal matrices, and $G / H$ is the space of symmetric unimodular unitary matrices. We consider now the cohomology of an arbitrary Klein space of a compact group in terms of differential forms; by an averaging process (as in $\$ \$ 12$ and 14) one can reduce all forms to invariant forms, where invariant means invariant under the action of the group. An invariant form is determined by its expression at any point $x_{0}$ (this leads at once to an inequality for the Betti numbers analogous to that of \$12). Let us call adjoint group of the space the group of linear transformations in the tangent space at $x_{0}$, induced by the elements of $G$, which leave $x_{0}$ fixed (i.e. $G_{x_{0}}$, cf. $\$ 17$ ); the value at $x_{0}$ of an invariant form is then invariant under the adjoint group. The symmetric spaces now have the special property that, by a theorem of Cartan [13 (15); 25], invariant forms are automatically closed. This means that the $p$ th Betti number is equal to the number of independent invariant $p$-forms (modulo nothing), and, upon considering the values of the forms at $x_{0}$, that it equals the number of independent skew-symmetric tensors with $p$ indices, invariant under the adjoint group; in more sophisticated language, one has to determine how often the trivial representation occurs in the $p$ th exterior power of the adjoint group (cf. §12). As an example for the procedure, consider again the Grassmann manifold $M_{n, k}$, the manifold of $k$-planes in $n$-space, under the orthogonal group $O(n)$. The isotropy group is the direct product $O(k) \times O(n-k)$ (to keep a $k$-plane in itself, one can rotate in the plane, and also in the perpendicular $(n-k)$-plane). The adjoint group can be considered as operating on the vector space of all $k \times(n-k)$ matrices $M$; the element $\left(T_{k}, T_{n-k}\right)$ (where $\left(T_{k} \in O(k)\right.$, $\left.T_{n-k} \in O(n-k)\right)$ maps $M$ into $T_{k} M T_{n-k}^{\prime}$. One considers now the representation of $G L(k) \times G L(n-k)$, given by the same formula. The complete reduction of the $p$ th exterior power of this representation has been given by Ehresmann [34]; one has to make use of the known theory of the representations of $G L(n)$, in particular the Young diagrams and the weights. For each irreducible constituent obtained, Iwamoto [51], using the known theory of representations of the orthogonal groups, determined what happens when one now restricts oneself to the orthogonal groups. One arrives at a fairly simple rule 
for the appearance of trivial representations or linear invariants, expressing the $p$ th Betti number in terms of partitions of $p$. The procedure and the results are similar for all other symmetric spaces; Iwamoto treated also the classical groups themselves in this way. That the algebraic methods of $\$ 22$ can be applied successfully to symmetric spaces has been mentioned already. Among other things this has led to an explicit formula for the Poincaré polynomials of the Grassmann manifolds.

25. Subgroups of maximum rank. Another class of (compact) coset spaces which was already mentioned in $\$ 18$, and $\$ 22$, consists of those spaces for which the isotropy groups are of maximum rank; according to $\$ 18$ they are just the coset spaces of positive characteristic. It is easy to see that any such space is topological product of coset spaces of the same type of simple Lie groups, thus reducing the problem of classifying these spaces considerably. Wang $[125 ; 126]$ and Borel and de Siebenthal $[5 ; 6]$ have given a complete list of the subgroups of maximal rank of the classical groups, and (Borel and de Siebenthal) of the maximal subgroups of maximal rank of the exceptional groups. Naturally this analysis makes very explicit use of what one knows about the Lie algebras of the simple groups. (In the treatment of Borel and de Siebenthal a characterization of connected subgroups of maximal rank is taken as the starting point of the classification: such groups are the components of $e$ of the normalizers of their own center.) As an example of the results: for the unitary unimodular group $S U(n)$ the subgroups in question are obtained by stringing groups of the type $U(k)$ (=group of unitary matrices of degree $k$ ) with properly chosen $k$-values along the diagonal and requiring the matrices so obtained to be unimodular. Out of the classification of these Klein spaces, with the help of the theory of a group modulo a maximal abelian subgroup (\$18), one gets the characteristic; the Betti numbers and the ring structure are not obtained here. By the way, subgroups of maximum rank are automatically closed, due to a theorem of Malcev of $\S 8$.

Along these lines it has been shown: If a (simply connected) Klein space has characeristic 1 , it is a single point [127]; if it has characteristic 2 , it is a sphere; if it has characteristic a prime $>2$, it is one of a small list of spaces $[7 ; 127]$.

26. Two-dimensional Klein spaces. Local groups of transformations. A very natural problem arising here is that of determining all two-dimensional Klein spaces. Completing earlier results in which the Klein bottle had been overlooked, Mostow [93] showed that 
exactly the following surfaces appear (noncompact groups allowed): plane, cylinder, torus, sphere, (open) Möbius strip, projective plane, Klein bottle. He arrived at this result by showing that for a coset space $G / H$ with $\operatorname{dim} G-\operatorname{dim} H=2$ the fundamental group has to be of a certain restricted type, which, by the classification of surfaces, admitted only the surfaces listed, and then showing explicitly that they actually are Klein spaces. His results include more, namely a complete classification (up to isomorphism) of all transitive Lie groups of transformations of surfaces.

The results mentioned in $\$ 8$ on the closedness of subgroups also have a significance for Klein spaces: they relate to the problem of extending a local transformation group to a (global) transformation group. "Local transformation group" means roughly that in the definition of homogeneous space of $\$ 17$, the $T_{\theta}$ are defined only for the elements $g$ in a neighborhood of $e$, and that they act only on some open subset of $W$; transitivity has to be defined properly. In an extension $G$ and $W$ may have to be replaced by locally equivalent objects. Starting from the simple observation that for a Klein space the isotropy group is always closed, it is shown [38; 93] that the extension problem amounts to determining which subalgebras of the Lie algebra of $G$ (or which "local subgroups") give rise to closed subgroups in the simply connected form $\tilde{G}$. In particular, all transitive local groups on an open set in $E^{k}, k<5$, can be extended to a group, acting transitively on some manifold $M^{k}$.

27. A converse problem. A problem which goes so to speak in the reverse direction is the following: Given a Klein space $W$, which groups (besides the one which defines $W$ ) can act transitively on $W$ ? (We assume the group to be effective, i.e., only the unit $e$ gives the identity transformation of $W$, and compact.) It is possible to answer this question completely when $W$ is a sphere $[7 ; 8 ; 89]$. To state the result: Except for three cases only those groups can act transitively on a sphere which are well known to do so, namely for even dimensions only the orthogonal group (plus $G_{2}$, which has $S^{6}$ as Klein space), for dimension $4 n+1$ the orthogonal, the unimodular unitary, and the unitary group, for dimension $4 n-1$ also the symplectic group and the full quaternion-unitary group (plus $S^{15}=\widetilde{B} 4 / \widetilde{B} 3$ and $S^{7}=\widetilde{B}_{3} / G_{2}$ ). The proofs depend on the general theorems on groups acting transitively on spheres (cf. \$21), plus the knowledge of the Betti numbers of the classical groups. To indicate a simple case: A symplectic group can not act transitively on $S^{4 n+1}$ since otherwise, by the theorem of $\$ 21$, it would have a primitive cocycle of dimension $4 n+1$, which it doesn't by the results of $\S 11$. The same problem has 
been treated by Matsushima [84] under the weaker assumption that $W$ is a homology sphere, i.e., that its Betti numbers equal those of a sphere, of odd dimension. The results can be described by saying that, up to a small, finite number of cases, which remain undecided, any such space is either actually a sphere or-and this is the only new case-the space of unit tangent vectors to a sphere of even dimension $(=O(2 n+1) / O(2 n-1)$; with the notation of $\$ 30$ we can write it as $\left.V_{2 n+1,2}\right)$, and that the groups act in standard fashion. The methods are similar to those indicated above; much stronger use is made of the Lie algebras of the groups involved. An important role is played by a theorem of Kudo which says that under the stated assumption on $W$ the isotropy group $H$ is not $\sim 0$ in $G$; this is also contained in Koszul's results ( $\$ 22)$.

For spheres of even dimension another approach has been given by A. Borel [7]: the sphere has characteristic two, and two is a prime number; this implies easily that the isotropy group must be a maximal subgroup of maximal rank, and now one can make use of the complete list of such groups in $\$ 25$.

A similar result is that any group acting transitively and effectively on the torus $T^{n}$ is actually $T^{n}$ itself $[7 ; 90]$. For one of the proofs one shows that because of the size of the Betti numbers of $T^{n}$, i.e., because of the large number of invariant differential forms, the isotropy group has to reduce to the unit element (or at any rate be finite); this implies that the group is homeomorphic with $T^{n}$, and therefore, using the known structure of compact groups $(\$ 10)$, is isomorphic with $T^{n}$.

28. Two-point homogeneous spaces. A recent result of $\mathrm{H}$. C. Wang [128] belongs to this group of problems. The question was to determine all compact (connected) metric spaces, which are two-point homogeneous in the sense that, for any four points $a, b, c, d$ with $\rho(a, b)=\rho(c, d)$, there exists an isometry sending $a$ into $c$ and $b$ into $d$. Wang showed by a simple ingenious argument that the group of isometries cannot have arbitrarily small normal subgroups, and therefore must be a (compact) Lie group, and the space is therefore a Klein space (of finite dimension, say $n$ ). The two-point condition implies that the isotropy group must be transitive on the directions issuing from a point, or in other words, that it is transitive over the $(n-1)$-sphere, and one can then apply the results described in $\$ 27$. One obtains a complete determination of all possibilities; the spaces occurring are: for odd $n$ only the spheres and projective spaces, for even $n$ there are in addition complex projective spaces, quaternion 
projective spaces, and the (16-dimensional) projective plane over the Cayley numbers.

29. Noncompact Klein spaces. Not too much is to be said about noncompact Klein spaces. Cartan showed [13 (15)] that the symmetric Riemannian spaces, whose groups of isometries are noncompact simple Lie groups, are all homeomorphic to $E^{n}$. He showed actually that in the group there exists a set of elements, called transvections (which behave somewhat like translations in Euclidean space), which set is homeomorphic with $E^{n}$, and whose elements are in 1:1 correspondence with the points of the symmetric space (under the natural projection). Incidentally, all these spaces have negative or zero Riemannian curvature (whereas closed symmetric spaces have non-negative curvature).

The analogue of the Malcev-Iwasawa theorem does not hold in general; there are spaces which are not Cartesian product of a compact space and an $E^{n}$. An example is given by one of the two sets complementary to a hyperquadric in (real or complex) projective space. Factorization does hold in the special case of a Klein space of a nilpotent Lie group $[82 ; 85]$ (a Lie algebra $L$ and its group are nilpotent if the sequence $L, L_{1}=[L, L], L_{2}=\left[L, L_{1}\right]$, ‥ ends with 0$)$. For the proof one can start from the fact that the (simply connected) group is homeomorphic with $E^{n}(\S 8)$, and that it is possible to give a description of the discrete subgroups (each such subgroup has the geometric structure of a lattice in $E^{n}$, but is in general noncommutative). Chevalley has shown (not yet published) that factorization (into a torus and a Euclidean space) holds for Klein spaces of solvable groups, provided the isotropy group is connected. Some new results have recently been announced by Mostow [94].

Nilpotent groups also provide some examples of compact Klein spaces, whose group is noncompact (the isotropy group, which by $\$ 18$ must be nonconnected, is here even discrete) [85]. The results obtained concern the first and second rational cohomology group; in particular the first Betti number is found to equal $\operatorname{dim} L-\operatorname{dim} L^{\prime}$, generalizing the fact that for the torus $T^{n}$ the first Betti number equals $n$.

30. Homotopy properties. As a last class of topological properties we should like to mention briefly the homotopy properties; this means principally the question of the homotopy groups [the $k$ th homotopy group $\pi_{k}(G)$ is the group of continuous maps of a $k$-sphere $S^{k}$ into $G$, or rather of classes of maps which can be deformed continuously into each other, under a certain addition, generalizing the 
elements and the operation of the fundamental group $\pi_{1}(G)$ ] (cf. [116] for this whole section).

That the fundamental group of a group is always abelian and that it is finite for compact semi-simple groups was mentioned earlier. It was also known long ago [13] that the second homotopy group $\pi_{2}(G)$ of a Lie group $G$ is always zero, which means that every continuous map of an $S^{2}$ into the group can be deformed into a single point (this follows from the construction at the end of $\$ 19$, since the image of a 2-sphere can always be so deformed as to not meet the $(n-3)$-dimensional set of singular elements). For coset spaces the situation is different, as the space $S^{2}$ itself shows. This sheds some light on the theorem of Malcev and Iwasawa (\$8) on the simple connectedness of a normal subgroup $N$ of a simply connected group $G$ : We consider the exact homotopy sequence of $G$ and $N$ and the fibre space isomorphism of $\pi_{2}(G / N)$ with the relative group $\pi_{2}(G ; N)$ [116]. Since $\pi_{1}(G)$ and $\pi_{2}(G)$ vanish, it follows that $\pi_{1}(N)$ is isomorphic with $\pi_{2}(G / N)$, even if $N$ is not normal; if $N$ is normal, then $\pi_{2}(G / N)$ is zero, and so is then $\pi_{1}(N)$. The results on higher homotopy groups are far from complete; what is known is in very close connection to what is known about the homotopy groups of spheres; the reason for this is that most of the existing results are obtained for groups acting transitively on spheres, by fibre space relations $[29 ; 30 ; 126 ; 133 ; 134 ; 136 ; 116]$. A special result of Pontryagin's [100] says that $\pi_{4}\left(A_{n}\right)=0$, for $n \geqq 2$; this is of interest because it shows that $A_{n}$ is not Cartesian product of $S^{3}$ with another space, whereas the homology properties would allow it.

Homotopy properties of Lie groups also play a role for the theory of fibre bundles. One particular problem relates to the so-called classifying or universal bundles. For a group $G$, a (principal) fibre bundle $B$ with fibre $G$ is called $n$-classifying or $n$-universal if it is arcwise connected and the homotopy groups $\pi_{i}(B)$ vanish for $1 \leqq i<n$. (The bundles, which have $G$ as transformation group of their fibre, and which have a polyhedron $X$ of dimension $<n$ as base space, are then in $1: 1$ correspondence with the homotopy classes of maps of $X$ into the base space of $B$, whence the term classifying [116;137].) Now, if $G$ is a compact Lie group, it is easy to construct such a bundle for any $n: G$ can be considered as subgroup of some orthogonal group $O(k)$ (since it has a faithful representation). We form now $O(n+k) / O(n) \times I_{k}$ (where $O(n) \times I_{k}$ means the elements of $O(n+k)$ whose last $k$ diagonal elements are 1$)$; this is a fibre bundle over $O(n+k) / O(n) \times G$, with fibre $O(n) \times G / O(n) \times I_{k}=G$ (cf. $\$ 17$ ). Moreover its homotopy groups $\pi_{i}$ vanish for $1 \leqq i<n$; for $k=1$ this 
follows from the fact that $O(n+1) / O(n) \times I_{1}$ is nothing else but the sphere $S^{n}$; for larger $k$ it follows similarly, by considering the chain $O(n+k), O(n+k-1), \cdots, O(n)$. An interesting example is obtained for $G=O(k)$, the case of sphere bundles, where the fibre is a $(k-1)$ sphere; the base space $O(n+k) / O(n) \times O(k)$ of the universal bundle can then be identified with the space of $(k-1)$-spheres on a $(n+k-1)$ sphere considered by Whitney [137], or equivalently with the Grassmann manifold $M_{n+k, k}$ of $k$-planes in $(n+k)$-space. Knowledge of the homology properties of $M_{n+k, k}$ is then of obvious importance, since for sphere bundles, by the classification theorem above, one has to consider maps of a space $X$ into $M_{n+k, k}$; such a map induces a homomorphism of the cohomology ring of $M_{n+k, k}$ into that of $X$, which depends only on the bundle corresponding to $f$; the image ring is called the characteristic ring of the bundle. (An algebraic counterpart has been mentioned in $\$ 22$.)

The manifold $O(m) / O(m-k) \times I_{k}$, which appeared here, can also be described as the space, each point of which is a system of $k$ mutually orthogonal unit vectors at the origin in Euclidean $m$-space (a $k$-frame); it is called the Stiefel manifold $V_{m, k}$. The $V_{m, k}$ were introduced by Stiefel [120] in connection with the problem of constructing over a manifold $M^{m}$ a system of $k$ vector fields, mutually orthogonal at each point of $M^{m}$. One is led, in obstruction theory [116], to a characteristic cocycle, which measures the degree of impossibility of the construction of the vector fields. The dimension of the cocycle is the first dimension for which the homotopy groups of $V_{m, k}$ are not zero plus one and the coefficients are taken exactly from this group. As indicated above, the critical dimension for $V_{m, k}$ is $m-k$; the corresponding groups $\pi_{m-k}\left(V_{m, k}\right)$ are known [116]. To quote the result: they are infinite cyclic if $m-k$ is even or $k=1$, of order two if $m-k$ is odd and $k>1$.

One special, but important, case is the following: We have seen that the sphere $S^{n}$ is the coset space of the orthogonal group $O(n+1)$ by the subgroup $O(n)$. Does there exist a map $f$ of $S^{n}$ into $O(n+1)$, such that $f$ followed by the projection of $O(n+1)$ onto $O(n)$ is the identity? Phrased differently: does there exist a continuous family of rotations, simply transitive over the sphere; or again, does the decomposition of $O(n+1)$ into cosets of $O(n)$ admit a cross section, i.e., a surface, which meets each coset of $O(n)$ in exactly one point? If a cross section existed, then, as one sees easily, $O(n+1)$ would be the Cartesian product of $O(n)$ and $S^{n}$, and, more interesting, there would exist $n$ everywhere independent vector fields, tangent to $S^{n}$, i.e. in the language of topology, $S^{n}$ would admit a parallelism. The 
obvious approach to this is to construct a cross section by starting at an arbitrary point of $O(n+1)$, expanding it gradually and studying the singularity or obstruction, into which one runs after a while [116]. We only state some results: It is an old story that spheres of even dimension do not admit a single (nonzero) vector field. B. Eckmann and G. W. Whitehead have shown that spheres of dimension $4 n+1$ admit only one independent vector field [29;133]; for spheres of dimension $4 n-1$ there exist more than one field, constructed e.g. with quaternions and Cayley numbers. Recently Steenrod and J. H. C. Whitehead [119] have shown, with the help of new homology invariants, that at most the spheres of dimension $2^{k}-1$ admit a parallelism (the only cases where a parallelism is known are $S^{1}, S^{3}$, and $S^{7}$, corresponding to complex numbers, quaternions, and Cayley numbers (cf. also [86])).

\section{BIBLIOGRAPHY}

Instead of listing all of Cartan's papers, we note that a complete list (up to 1939) appears in [14] and that the papers most relevant to the subject are cited in the bibliography to [13]; we shall refer to these papers by the notation $[13(n)]$. The bibliography contains, besides the papers referred to in the text, a number of other papers of direct interest for the topics discussed. [116] contains a bibliography on fibre bundles.

1. M. Abe, Sur la métrique riemannienne et l'élément de volume dans les espaces de groupes de Lie, Proc. Imp. Acad. Tokyo vol. 19 (1943) pp. 629-634.

2. P. S. Alexandroff and H. Hopf, Topologie I, Berlin, 1935.

3. G. Birkhoff, Lie groups isomorphic with no linear group, Bull. Amer. Math. Soc. vol. 42 (1936) pp. 883-888.

4. - Representability of Lie algebras and Lie groups by matrices, Ann. of Math. vol. 38 (1937) pp. 526-532.

5. A. Borel and J. de Siebenthal, Sur les sous-groupes fermés connexes de rang maximum des groupes de Lie clos, C. R. Acad. Sci. Paris vol. 226 (1948) pp. 1662-1664.

6. - Les sous-groupes fermés connexes de rang maximum des groupes de Lie clos, Comment. Math. Helv. vol. 23 (1949-1950) pp. 200-221.

7. A. Borel, Some remarks about transformation groups transitive on spheres and tori, Bull. Amer. Math. Soc. vol. 55 (1949) pp. 580-586.

8. - Le plan projectif des octaves et les sphères comme espaces homogènes, C. R. Acad. Sci. Paris vol. 230 (1950) pp. 1378-1380.

9. - Sur la cohomologie des espaces fibrés principaux et des espaces de groupes de Lie compacts, to appear in Ann. of Math.

9a. - Impossibilitê de fibrer une sphère par un produit de sphères, C.R. Acad. Sci. Paris vol. 231 (1950) pp. 943-945.

9b. - Sur la cohomologie des variêtes de Stiefel et de certaines groupes de Lie, C.R. Acad. Sci. Paris vol. 232 (1951) pp. 1628-1630. 
9c. - La transgression dans les espaces fubrés principaux, C. R. Acad. Sci. Paris vol. 232 (1951) pp. 2392-2394.

9d. - Sur la cohomologie des espaces homogènes des groupes de Lie compacts, C.R. Acad. Sci. Paris vol. 233 (1951) pp. 569-571.

9e. A. Borel and J.-P. Serre, Détermination des p-puissances réduites de Steenrod dans la cohomologie des groupes classiques. Applications, C.R. Acad. Sci. Paris vol. 233 (1951) pp. 680-682.

10. R. Brauer, Sur les invariants intégraux des variétés des groupes de Lie simple clos, C. R. Acad. Sci. Paris vol. 201 (1935) pp. 419-421.

11. L. Calabi and C. Ehresmann, Sur les extension des groupes topologiques, C. R. Acad. Sci. Paris vol. 229 (1949) pp. 1551-1553.

12. L. Calabi, Sur les extensions des groupes topologiques, C. R. Acad. Sci. Paris vol. 229 (1949) pp. 413-415.

13. E. Cartan, La topologie des groupes de Lie, Acutalités Scientifiques et Industrielles, no. 358, Paris, 1936, also L'Enseignement Mathématique vol. 35 (1936) pp. $177-200$.

14. - - Selecta, Jubile Scientifique, Paris, Gauthier-Villars, 1939.

15. H. Cartan, Sur la cohomologie des espaces où opère un groupe. Notions algebriques preliminaires, C. R. Acad. Sci. Paris vol. 226 (1948) pp. 148-150.

16. - Notions d'algèbre diffêrentielle; application aux groupes de Lie et aux variêtés où opère un groupe de Lie, Colloque de topologie (espaces fibrés), Bruxelles, 1950; Liège and Paris, 1951.

17. - La transgression dans un groupe de Lie et dans un espace fibré principal, Colloque de topologie (espaces fibrés), Bruxelles, 1950; Liège and Paris, 1951.

18. S. S. Chern, A simple intrinsic proof of the Gauss-Bonnet formula for closed Riemannian manifolds, Ann. of Math. vol. 45 (1944) pp. 747-752.

19. - On the multiplication in the characteristic ring of a sphere bundle, Ann. of Math. vol. 49 (1948) pp. 362-372.

20. - Differential geometry of fiber bundles, to appear.

21. C. Chevalley, Theory of Lie groups I, Princeton, 1946.

22. - On the topological structure of solvable groups, Ann. of Math. vol. 42 (1941) pp. 668-675.

23. - Two theorems on solvable topological groups, Lectures in Topology, Ann Arbor, 1941.

24. - - An algebraic proof of a property of Lie groups, Amer. J. Math. vol. 63 (1941) pp. 785-793.

25. C. Chevalley and S. Eilenberg, Cohomology theory of Lie groups and Lie algebras, Trans. Amer. Math. Soc. vol. 63 (1948) pp. 85-124.

26. C. Chevalley, Determination of the Betti numbers of the exceptional Lie groups, Proceedings of the International Congress of Mathematicians, 1950 (to appear).

27. D. van Dantzig, and B. L. van der Waerden, Über metrisch homogene Räume, Abh. Math. Sem. Hamburgischen Univ. vol. 6 (1928) pp. 367-376.

28. E. Dynkin, The structure of semi-simple algebras, Uspehi Matematiceskih Nauk (N. S.) vol. 2 (1947) pp. 59-127, translated in American Mathematical Society Translation no. $17,1950$.

29. B. Eckmann, Zur Homotopietheorie gefaserter Räume, Comment. Math. Helv. vol. 14 (1941-1942) pp. 141-192.

30. - Über die Homotopiegruppen von Gruppenräumen, Comment. Math. Helv. vol. 14 (1941-1942) pp. 234-256.

31. - Systeme von Richtungsfeldern in Spharen, Comment. Math. Helv. vol. 15 (1942-1943) pp. 1-26. 
32. C. Ehresmann, Les invariants integraux et la topologie de l'espace projectif regle, C.R. Acad. Sci. Paris vol. 194 (1932) pp. 2004-2006.

33. - Sur la topologie de certaines variêtes algébriques, C.R. Acad. Sci. Paris vol. 196 (1933) pp. 152-154.

34. - Sur la topologie de certains espaces homogènes, Ann. of Math. vol. 35 (1934) pp. 396-443.

35. - Sur la topologie de certaines variêtés algébriques rélles, J. Math. Pures Appl. vol. 16 (1937) pp. 69-110.

36. — Sur la topologie des groupes simples clos, C.R. Acad. Sci. Paris vol. 208 (1939) pp. 1263-1265.

37. - Sur la variêté des gênératrices planes d'une quadrique réelle et sur la topologie du groupe orthogonal d $n$ variables, C.R. Acad. Sci. Paris vol. 208 (1939) pp. 321-323.

38. - Sur les espaces localement homogènes, L'Enseignement Mathématique vol. 35 (1936) pp. 317-333.

39. F. Gantmacher, On the classification of real simple Lie groups, Mat. Sbornik N.S. vol. 5 (1939) pp. 217-249.

40. M. Gotô, Faithful representations of Lie groups I, Mathematica Japonicae vol. 1 (1949) pp. 107-119.

41. - Faithful representations of Lie groups II, Nagoya Math. J. vol. 1 (1950) pp. 91-107.

42. Harish-Chandra, On faithful representations of Lie groups, Proceedings of the American Mathematical Society vol. 1 (1950) pp. 205-210.

43. - Lie algebras and the Tannaka duality theorem, Ann. of Math. vol. 51 (1950) pp. 299-330.

44. - On the radical of a Lie algebra, Proceedings of the American Mathematical Society vol. 1 (1950) pp. 14-17.

45. H. Hopf, Sur la topologie des groupes clos de Lie et de leurs genéralisations, C.R. Acad. Sci. Paris vol. 208 (1939) pp. 1266-1267.

46. - - Über die Topologie der Gruppen-Mannigfaltigkeiten und ihrer Verallgemeinerungen, Ann. of Math. vol. 42 (1941) pp. 22-52.

47. - Über den Rang geschlossener Lie'scher Gruppen, Comment. Math. Helv. vol. 13 (1940-1941) pp. 119-143.

48. - Maximale Toroide und singuläre Elemente in geschlossenen Lie'schen Gruppen, Comment. Math. Helv. vol. 15 (1942-1943) pp. 59-70.

49. H. Hopf and H. Samelson, Ein Satz ulber die Wirkungsräume geschlossener Lie'scher Gruppen, Comment. Math. Helv. vol. 13 (1940-1941) pp. 240-251. 1941.

50. W. V. D. Hodge, The theory and applications of harmonic integrals, Cambridge,

51. H. Iwamoto, On integral invariants and Betti numbers of symmetric Riemannian spaces I, II, J. Math. Soc. Jap. vol. 1 (1949) pp. 91-110, 235-243.

52. K. Iwasawa, On some types of topological groups, Ann. of Math. vol. 50 (1949) pp. $507-558$.

53. Y. Kawada, On the invariant differential forms of local Lie groups, J. Math. Soc. Jap. vol. 1 (1949) pp. 217-225.

54. J. L. Koszul, Sur la troisième nombre de Betti des espaces de groupes de Lie compacts, C.R. Acad. Sci. Paris vol. 224 (1947) pp. 251-253.

55. - Sur les opérateurs de derivation dans un anneau, C.R. Acad. Sci. Paris vol. 225 (1947) pp. 217-219.

56. — - Sur l'homologie des espaces homogènes, C.R. Acad. Sci. Paris vol. 225 (1947) pp. 477-479. 
57. - Sur l'homologis et la cohomologie des algèbres de Lie, C.R. Acad. Sci. Paris vol. 228 (1949) pp 288-290.

58. - - Sur la cohomologie relative des algèbres de Lie, C.R. Acad. Sci. Paris vol. 228 (1949) pp. 457-459.

59. - Homologie et cohomologie des algèbres de Lie, Bull. Soc. Math. France vol. 78 (1950) pp. 65-127.

60. - - Sur un type d'algèbres différentielles en rapport avec la transgression, Colloque de topologie (espaces fibrés), Bruxelles, 1950; Liège and Paris, 1951.

61. S. Lefschetz, Algebraic topology, Amer. Math. Soc. Colloquium Publications, vol. 27, New York, 1942.

62. J. Leray, Sur la forme des espaces topologiques et sur les points fixes des reprélentation, J. Math. Pures Appl. vol. 54 (1945) pp. 95-167.

63. - L'anneau d'homologie d'une reprêsentation, C.R. Acad. Sci. Paris vol. 222 (1946) pp. 1366-1368.

64. ——, Structure de l'anneau d'homologie d'une représentation, C.R. Acad. Sci. Paris vol. 222 (1946) pp. 1419-1422.

65. - Propriétés de l'anneau d'homologie de la projection d'un espace fibré sur sa base, C.R. Acad. Sci. Paris vol. 223 (1946) pp. 395-397.

66. - - Sur l'anneau d'homologie de l'espace homogène, quotient d'un groupe clos par un sous-groupe abélien connexe maximum, C.R. Acad. Sci. Paris vol. 223 (1946) pp. 412-415.

67. - Espace où opère un groupe de Lie compact et connexe, C.R. Acad. Sci. Paris vol. 228 (1949) pp. 1545-1547.

68. - A Aplication continue commutant avec les êlements d'un groupe de Lie compact, C.R. Acad. Sci. Paris vol. 228 (1949) pp. 1784-1786.

69. - Détermination, dans les cas non-exceptionnels, de l'anneau de cohomologie de l'espace homogène quotient d'un groupe de Lie compact par un sous-groupe de même rang, C.R. Acad. Sci. Paris vol. 228 (1949) pp. 1902-1904.

70. - Sur l'anneau de cohomologie des espaces homogènes, C.R. Acad. Sci. Paris vol. 229 (1949) pp. 281-283.

71. - - Sur l'homologie des groupes de Lie, des espaces homogènes et des espaces fibrés principaux, Colloque de topologie (espaces fibrés), Bruxelles, 1950; Liège and Paris, 1951.

72. - L'anneau spectral et l'anneau fibrê d'homologie d'un espace localement compact et d'une application continue, J. Math. Pures Appl. vol. 29 (1950) pp. 1-139.

73. - L'homologie d'un espace fibré dont la fibre est connexe, J. Math. Pures Appl. vol. 29 (1950) pp. 169-213.

74. A. Malcev, On the simple connectedness of invariant subgroups of Lie groups, C.R. (Doklady) Acad. Sci. URSS vol. 34 (1942) pp. 10-13.

75. - Subgroups of Lie groups in the large, C.R. (Doklady) Acad. Sci. URSS. vol. 36 (1942) pp. 5-7.

76. - - On the decomposition of an algebra in direct sum of their radical and semi-simple subalgebra, C.R. (Doklady) Acad. Sci. URSS. vol. 36 (1942) pp. 46-50.

77. - On the structure of Lie groups in the large, C.R. (Doklady) Acad. Sci. URSS. vol. 37 (1942) pp. 3-5.

78. —- On linear Lie groups, C.R. (Doklady) Acad. Sci. URSS. vol. 40 (1943) pp. 87-89.

79. - On semi-simple subgroups of Lie groups in the large, Bull. Acad. Sci. URSS. Sér. Math. vol. 8 (1944) pp. 143-174. 
80. - On the theory of the Lie groups in the large, Mat. Sbornik N.S. vol. 16 (1945) pp. 163-189.

81. - Corrections to my paper "On the theory of Lie groups in the large," Mat. Sbornik N.S. vol. 19 (1946) pp. 523-524.

82. - On a class of homogeneous spaces, Izvestiya Akademii Nauk SSSR. Sér. Math. vol. 13 (1949) pp. 9-32, also Amer. Math. Soc. Translation no. 42 (1951).

83. Y. Matsushima, On the faithful representation of Lie groups, J. Math. Soc. Jap. vol. 1 (1949) pp. 254-261.

84. - On a type of subgroups of compact Lie group, Nagoya Math. J. vol. 2 (1951) pp. 1-15.

85. - On the discrete subgroups and homogeneous spaces of nilpotent Lie groups, Nagoya Math. J. vol. 2 (1951) pp. 95-110.

86. C. E. Miller, The topology of rotation groups, Chicago dissertation, 1951, to appear in Ann. of Math.

87. D. Montgomery and L. Zippin, Topological transformation groups I, Ann. of Math. vol. 41 (1940) pp. 778-791.

88. - - A theorem on Lie groups, Bull. Amer. Math. Soc. vol. 48 (1942) pp. $448-452$.

89. D. Montgomery and H. Samelson, Transformation groups of spheres, Ann. of Math. vol. 44 (1943) pp. 454-470.

90. - Groups transitive on the n-dimensional torus, Bull. Amer. Math. Soc. vol. 49 (1943) pp. 455-456.

91. D. Montgomery, Simply connected homogeneous spaces, Proceedings of the American Mathematical Society vol. 1 (1950) pp. 467-469.

92. G. D. Mostow, A new proof of E. Cartan's theorem on the topology of semi-simple groups, Bull. Amer. Math. Soc. vol. 55 (1949) pp. 969-980.

93. - - The extendability of local Lie groups of transformations and groups on surfaces, Ann. of Math. vol. 52 (1950) pp. 606-637.

94. - On the topology of non-compact Klein spaces, Bull. Amer. Math. Soc. Abstract 57-2-177.

95. J. Nordon, Les éléments d'homologie des quadriques et des hyperquadriques, Bull. Soc. Math. France vol. 74 (1946) pp. 11-129.

96. L. Pontryagin, Topological groups, Princeton, 1939.

97. - On Betti numbers of compact Lie's groups, C.R. (Doklady) Acad. Sci. URSS. vol. 1 (1935) pp. 433-437.

98. - Sur les nombres de Betti des groupes de Lie, C.R. Acad. Sci. Paris vol. 200 (1935) pp. 1277-1280.

99. - Homologies in compact Lie groups, Mat. Sbornik N.S. vol. 6 (1939) pp. $389-422$.

100. - Über die topologische Struktur de Lie'schen Gruppen, Comment. Math. Helv. vol. 13 (1940-1941) pp. 227-238.

100a. - Characteristic cycles on differentiable manifolds, Mat. Sbornik N.S. vol. 21 (1947) pp. 233-284.

100b. - Some topological invariants of closed Riemannian manifolds, Izvestiya Akad. Nauk SSSR. Sér. Mat. vol. 13 (1949) pp. 125-162.

101. G. Racah, Sulla caratterizzazione delle rappresentazioni irreducibili dei gruppi semisemplici di Lie, Rend. d. Accad. dei Lincei vol. 8 (1950) pp. 108-112.

102. G. de Rham, Sur l'analysis situs des variétés à $n$ dimensions, J. Math. Pures Appl. vol. 10 (1931) pp. 115-200. 
103. H. Samelson, Über die Sphären, die als Gruppenräume auftreten, Comment. Math. Helv. vol. 13 (1940-1941) pp. 144-155.

104. - Beiträge zur Topologie der Gruppen-Mannigfaltigkeiten, Ann. of Math. vol. 42 (1941) pp. 1091-1137.

105. —_, A note on Lie groups, Bull. Amer. Math. Soc. vol. 52 (1946) pp. 870-873.

106. - Sur les sous-groupes de dimension trois des groupes de Lie compacts, C.R. Acad. Sci. Paris vol. 228 (1949) pp. 630-631.

107. O. Schreier, Abstrakte kontinuierliche Gruppen, Abh. Math. Sem. Hamburgischen Univ. vol. 4 (1925) pp. 233-244.

108. - Die Verwandtschaft stetiger Gruppen im Grossen, Abh. Math. Sem. Hamburgischen Univ. vol. 5 (1926) pp. 15-32.

109. H. Seifert and W. Threlfall, Lehrbuch der Topologie, Leipzig, 1934.

110. A. S. Shapiro, Group extensions of compact Lie groups, Ann. of Math. vol. 50 (1949) pp. 581-586.

111. R. Schizuma, Über die Untergruppen geschlossener Lie'scher Gruppen, Proc. Imp. Acad. Tokyo vol. 20 (1944) pp. 670-673.

112. J. de Siebenthal, Sur certaines sous-groupes de rang un des groupes de Lie clos, C.R. Acad. Sci. Paris vol. 230 (1950) pp. 910-912.

113. P. A. Smith, Properties of group manifolds, Proc. Nat. Acad. Sci. U.S.A. vol. 17 (1933) pp. 612-618.

114. - The fundamental group of a group manifold, Ann. of Math. vol. 36 (1935) pp. 210-227.

115. - The topology of transformation groups, Bull. Amer. Math. Soc. vol. 44 (1938) pp. 497-514.

116. N. E. Steenrod, The topology of fiber bundles, Princeton, 1951.

117. - The classification of sphere bundles, Ann. of Math. vol. 45 (1944) pp. 294-311.

118. N. E. Steenrod and A. W. Tucker, Real n-quadrics as sphere bundles, Bull. Amer. Math. Soc. vol. 47 (1941) p. 399.

119. N. E. Steenrod and J. H. C. Whitehead, Vector fields on the $n$-sphere, Proc. Nat. Acad. Sci. U.S.A. vol. 37 (1951) pp. 58-62.

120. E. Stiefel, Richtungsfelder und Fernparallelismus in Mannigfaltigkeiten, Comment. Math. Helv. vol. 8 (1935-1936).

121. - - Über eine Beziehung zwischen geschlossenen Lie'schen Gruppen und diskontinuierlichen Bewegungsgruppen Euklidischer Räume und ihre Anwendung auf die Aufzählung der einfachen Lie'schen Gruppen, Comment. Math. Helv. vol. 14 (19411942) pp. 350-380.

122. - Kristallographische Bestimmung der Charaktere der geschlossenen Lie'schen Gruppen, Comment. Math. Helv. vol. 17 (1944-1945) pp. 165-200.

123. - Sur les nombres de Betti des groupes de Lie compacts, Colloques internationaux du Centre National de la Recherche, Paris, no. 12, Topologie algebrique, pp. 97-101.

124. B. L. van der Waerden, Die Klassifizierung der einfachen Lie'schen Gruppen, Math. Zeit. vol. 37 (1933) pp. 446-462.

125. H. C. Wang, Homogeneous spaces with non-vanishing Euler characteristic, Science Record. Academia Sinica vol. 2 (1949) pp. 215-219.

126. - Homogeneous spaces with non-vanishing Euler characteristic, Ann. of Math. vol. 50 (1949) pp. 915-953. 
127. - A new characterization of spheres of even dimension, Indagationes Mathematicae vol. 11 (1949) pp. 286-295 = Neder. Akad. Wetensch. vol. 52 (1949) pp. 838-847.

128. - Two-point homogeneous spaces, to appear in Ann. of Math.

129. A. Weil, Démonstration topologique d'un théorème fondamental de Cartan, C.R. Acad. Sci. Paris vol. 200 (1935) pp. 518-520; Mat. Sbornik N.S. vol. 1 (1936) p. 779.

130. H. Weyl, Theorie der Darstellung kontinuierlicher halb-einfacher Gruppen durch lineare Transformationen, I, II, III, Math. Zeit. vol. 23 (1925) pp. 271-309 and vol. 24 (1926) pp. 328-395.

131. - The classical groups, Princeton, 1946.

132. - - On the structure and representations of continuous groups I, II, mimeographed notes, Institute for Advanced Study, Princeton, 1933-1934, 1934-1935.

133. G. W. Whitehead, Homotopy properties of the real orthogonal groups, Ann. of Math. vol. 43 (1942) pp. 132-146.

134. - On the homotopy groups of spheres and rotation groups, Ann. of Math. vol. 43 (1942) pp. 634-640.

135. J. H. C. Whitehead, On the decomposition of an infinitesimal group, Proc. Cambridge Philos. Soc. vol. 32 (1936) pp. 229-237.

136. - On the groups $\pi_{r}\left(V_{n, m}\right)$ and sphere bundles, Proc. London Math. Soc. vol. 48 (1944) pp. 243-291; Corrigendum, ibid. vol. 49 (1947) pp. 479-481.

137. H. Whitney, Topological properties of differentiable manifolds, Bull. Amer. Math. Soc. vol. 43 (1937) pp. 785-805.

138. E. Witt, Spiegelungsgruppen und Aufzd̈hlung halb-einfacher Lie'scher Ringe, Abh. Math. Sem. Hamburgischen Univ. vol. 14 (1941) pp. 289-322.

139. Chih-Tah Yen, Sur les polynomes de Poincaré des groupes de Lie exceptionnels, C.R. Acad. Sci. Paris vol. 228 (1949) pp. 628-630.

140. - Sur les représentations linéaires de certains groupes et les nombres de Betti des espaces homogènes symétriques, C.R. Acad. Sci. Paris vol. 228 (1949) pp. 1367-1369.

141. L. Zippin, Transformation groups, Lectures in Topology, Ann Arbor, 1941.

UNIVERSITY OF MichigAN 